\title{
REMARQUES SUR LA SUBORDONNÉE TEMPORELLE A L'ÉPOQUE CLASSIQUE ET A L'ÉPOQUE TARDIVE, CHEZ JORDANES
}

Les oeuvres de Jordanès représentent, comme on le dit souvent, "une source inépuisable pour l'étude du latin vulgaire"1. Nous y ajoutons certains éclaircissements: le texte de Jordanès est inestimable pour la connaissance du latin tardif, vulgaire et cultivé.

Jordanès, d'origine ostrogothe, né et formé en Scythia Minor, quelque part en Dobroudja (province de l'Empire byzantin), est connu d'abord comme clerc d'un chef d'Alains, au début du $\mathrm{VI}^{\mathrm{e}}$ siècle (mais on ignore combien de temps exactement il eut cette charge), en Moesia Inferior, puis on le retrouve comme moine et fort probablement évêque d'une communauté gothique dans le nord de l'Italie. A part la langue maternelle, Jordanès avait encore appris la langue des Alains et, naturellement, le latin et le grec, nécessaires d'abord à sa fonction de notaire et à l'établissement des relations des Goths et des Alains avec l'Empire de Constantinople et, plus tard, à la formation même de Jordanès en tant qu'ecclésiastique et historien. Ce qui plus est: l'étude de ses oeuvres nous fait voir que l'historiographie de l'époque impériale romaine était familière à Jordanès et qu'il connaissait bien d'importants poètes comme Virgile et Lucain.

Des ouvrages de Jordanès, rédigés en Moesia et Italia, se sont conservés seulement deux: De origine actibusque gentis Romanorum, titre abrégé en Romana (titres imposés par l'édition de Th. Mommsen, dans la collection "Monumenta Germaniae historica", V: 1, Hannover, 1882; édition anastatique - Berlin, 1961) et De origine actibusque Getarum, titre abrégé: Getica (titres cités d'après la même édition de Th. Mommsen, ci-dessus mentionnée). ${ }^{2}$

1 Voir G. Popa-Lisseanu, Introduction à l'édition des Getica, dans "Fontes historiae Daco-Romanorum", vol. XIV, Bucarest, 1939, p. 9; voir en plus l'étude de A. Kappelmacher, dans Pauly-Wissowa, Real-Enzyclopädie der Klassischen Altertumswissenschaft, vol. IX, Stuttgart, 1916, p. 1925: "er (Iordanes) verwendet die wirklich lebende Sprache des gemeinen Mannes, wie die grosse Masse der zeitgenössischen Inschriften aufweist."; voir aussi l'opinion de Fr. Brunhölzl: "sein stark vulgär gefärbtes Latein." (Geschichte der lateinischen Literatur des Mittelalters, München, 1975, p. 30).

2 Dans notre étude, nous utiliserons seuls les titres abrégés Romana et Getica.

Pour ce qui est du titre du second ouvrage et de la confusion de Jordanès entre Goths et Gètes, voir R. Iordache, La confusion "Gètes - Goths" dans "Getica" de Jordanès, dans "Helmantica", XXXIV, Salamanque, 1983. 
Les ouvrages conservés, d'ailleurs les plus importants de Jordanès, sont en fait deux bréviaires, de l'histoire de Rome et, respectivement, de l'histoire des Goths.

De l'analyse des événements exposés en Romana et Getica nous arrivons à la conclusion que la réduction des deux oeuvres s'achevait pendant les derniers mois de l'année 550 et les premiers trois mois de l'année suivante, dans un puissant centre urbain d'Italie, vraisemblablement Ravenne. Il s'agit d'une époque historique spéciale, époque de grands troubles socio-politiques et militaires (parmi les événements importants rappelons la conquête de l'Italie par les armées des généraux Bélisaire et Narsès, au nom de l'empereur d'Orient - Justinien, victoire remportée sur les Ostrogoths du roi Totila).

Au point de vue linguistique, Romana et Getica présentent une intéressante combinaison de latin vulgaire (surtout du $\mathrm{VI}^{\mathrm{e}}$ siècle) et de latin de chancellerie (des juristes et ecclésiastiques) de l'époque tardive, sans qu'il y manque pour autant des éléments de pur latin classique.

Le degré de difficulté existant dans le texte de Jordanès est des plus élevés, surtout pour les chercheurs qui n'ont pas l'habitude du latin médiéval:

- c'est ainsi que dans les oeuvres de Jordanès apparaissent aussi bien des faits récents de latin vulgaire, que des faits plus anciens ou fort anciens de latin vulgaire, qui continuent de subsister dans le siècle de Jordanès (et qui se retrouvent dans une large mesure dans les langues romanes). Parmi les faits assez nouveaux de latin vulgaire, citons l'emploi de la locution tantum quod avec le sens d'“aussitôt que"; voir aussi la construction de la conjonction mox avec le plus-que-parfait du subjonctif; parmi les faits anciens, signalons l'emploi de postquam avec le subjonctif imparfait.

- Il existe aussi des aspects en quelque mesure opposés à ceux qu'on vient d'indiquer, en fait les divers éléments appartenant au style de chancellerie: l'usage de la conjonction quatenus et d'autres adverbes de la même famille; l'emploi bien répandu de quasi; 1 'usage de qualiter; etc. $^{3}$

- On pourrait parler, dans certains cas, de préférences marquées, relevant de la personnalité de l'auteur. C'est ainsi que le fréquent usage de la conjonction dum doit s'expliquer par une véritable prédilection de Jordanès pour cet adverbe. Il ne faut pas cependant perdre de vue le fait que cette conjonction était beaucoup employée dans le latin vulgaire et, d'autre part, que ce large usage tombait sous l'incidence de cette loi du latin vulgaire de l'époque tardive qui substituait aux conjonctions anciennes, usées et manquant d'expressivité, des formules d'expression bien plus précises. ${ }^{4}$

3 Voir, tant pour l'emploi de quatenus que pour celui de quasi, certaines données chez $\mathbf{R}$. Iordache, Observaciones sobre la subordinada causal en las obras de Jordanes, dans "Helmantica", XXVIr, Salamanque, 1976, pp. 51-52 et pp. 46-48.

4 Quant à l'usage de dum chez Jordanès, voir R. Iodache, Observaciones sobre la subordinada causal en las obras de Jordanes, op.cit., pp. 29-42. 
- On retrouve aussi des particularités propres au lieu où s'est formé l'auteur (Moesia Inferior): le large usage de in pour ad et aussi l'usage de in à la place de per. ${ }^{5}$

- Pour certaines dérogations aux normes du latin cultivé de l'époque classique on peut supposer l'influence du grec tardif: l'utilisation du participe futur à valeur finale etc. Il n'y manque pas non plus des éléments de pensée gothique.

- Assez fréquents sont les hyperurbanismes: absence des prépositions là où elles étaient absolument nécessaires, utilisation de l'imparfait du subjonctif pour le plus-que-parfait du subjonctif etc. ${ }^{6}$ Précisons que les hyperurbanismes apparaissent dans tous les compartiments de la grammaire, ainsi que dans le vocabulaire.

L'empressement que met l'auteur dans l'achèvement de la rédaction de ses oeuvres (ses efforts de mise au net des ouvrages précédant de peu la conquête de l'Italie par le général Narsès, période où Jordanès pouvait reprendre la question de l'importance de la fusion spirituelle et matérielle des Goths et des Romains) ${ }^{7}$ est, en bien des cas, à l'origine des erreurs d'inattention quant à la notation de la lettre finale, voire de l'omission de la lettre ou des lettres finales. L'imitation de certains historiens célèbres (Tacite, Ammien etc.) et, en outre, sa prétention de s'en distinguer, même de réaliser des phrases supérieures aux modèles (sur le plan de la correction et de l'élégance), ont fréquemment tourné à un confus accord d'idées, faisant leur part soit aux omissions, soit aux répétitions. Si l'on ajoute à tout cela les énumérations imprécises de tribus, de chefs de peuplades et tribus, de lieux de combats (qui, parfois, ne sont pas indiqués par d'autres historiens et qui sont impossibles à identifier jusqu'à ce jour), on aura, en grand, un aperçu des difficultés que pose le texte de Jordanès.

La plupart des phrases qu'on trouve dans les oeuvres de Jordanès comportent une multitude d'écarts par rapport aux règles du latin cicéronien. On pourrait dire, à juste raison, que dans nombre de ses phrases presque chaque mot comporte une faute, voire plusieurs, de nature différente, que ce soit au point de vue de la graphie, ou bien de la morphologie, de la syntaxe, du lexique, de l'ordre des mots dans la proposition et dans la phrase.

La plupart des dérogations aux normes du latin “d'or" s'expliquent par l'influence du latin vulgaire. Ces écarts sont non seulement très nombreux, mais encore de types très différents. La fréquence des types de fautes du texte de Jordanès correspond d'habitude à la fréquence des types respectifs d'erreurs dans le latin vulgaire de l'époque tardive (par exemple: le large usage de quia causal par rapport à quod causal;

$5 \quad$ Sur ce point, voir. R. Iordache, Elementos vulgares de la obra de Jordanes, dans "Helmantica", XXIV, Salamanque, 1973, pp. 132-33.

6 En ce qui concerne les hyperurbanismes des oeuvres de Jordanès, voir R. Iordache, Elementos vulgares de la obra de Jordanes, op.cit., p. 134; voir aussi R. Iordache, Observaciones sobre la subordinada causal en las obras de Jordanes, op.cit., pp. 9 et 54 . D'ailleurs la présentation ci-dessus des particularités linguistiques de Jordanès nous appartient entièrement.

7 Sur ce sujet, ainsi que sur les buts de la rédaction de Romana et de Getica, voir R. Iordache, $L a$ confusion Gètes" - Goths" dans "Getica" de Jordanès, op.cit. p. 319; pp. 321-23; note 2. 
l'usage du plus-que-parfait du subjonctif pour le plus-que-parfait de l'indicatif etc.). Il s'agit le plus souvent d'écarts qui se continuent dans les langues romanes.

Les oeuvres de Jordanès ne manquent pas cependant de passages correctement construits, selon toutes les règles du latin "d'or" (parfois copiés sur ses prédécesseurs). Les phrases, ou les membres de phrase (ces derniers surtout), rédigés correctement et même élégamment au point de vue stylistique, sont cependant rares par comparaison au nombre de phrases où abondent les dérogations aux normes du latin cultivé de l'époque classique.

Rappelons que les mots et les tournures vulgaires ne sont pas toujours de simples dérogations aux normes classiques. Assez souvent, leur rôle est d'assurer au texte un plus haut degré d'expressivité artistique; c'est aussi le cas des éléments appartenant au latin de chancellerie, d'autre part - des hyperurbanismes et, naturellement, des éléments proprement dits poétiques (de bonne qualité). ${ }^{8}$

L'étude des subordonnées temporelles dans les écrits de Jordanès est d'un intérêt tout spécial $^{9}$, vu les caractéristiques de l'utilisation des conjonctions temporelles et de leurs corrélatifs, ainsi que les aspects particuliers que présente l'emploi des modes et des temps dans les subordonnées et les principales en question. Certains aspects qui se détacheront relèvent de l'influence du latin vulgaire sur l'auteur, d'autres ressortent à sa personnalité, à sa manière propre d'utiliser le latin.

\section{LE RAPPORT DE SIMULTANÉITÉ}

Les propositions temporelles dont l'action était simultanée avec l'action de la principale étaient introduites en latin cultivé, à l'époque classique, par les conjonctions suivantes: dum explicatif-temporel ${ }^{10}$, donec, quoad et quamdiu; cum suivi de l'indicatif (que les grammaires appellent souvent cum "temporale" ou cum déterminatif, ou bien cum "temporel proprement dit" - en fait un dérivé de la valeur originelle de la conjonction cum, à savoir la valeur instrumentale-modale ${ }^{11}$ ), cum narratiuum ou

8 Jordanès connaisait d'ailleurs la recommandation de Cicéron (reprise ensuite par tous les grands historiens latins - notamment par Tite-Live et Tacite) de présenter l'histoire sous une forme littéraire-oratoire.

9 Les écrits de Jordanès ont été très peu étudiés jusqu'à présent. Même la dissertation de Fr. Werner (Die Latinität der "Getica" des Iordanes, Halle, 1908), considérée comme la plus importante étude sur les Getica de Jordanès, n'aborde pas d'une manière satisfaisante les divers aspects du latin de Jordanès. D'ailleurs, les problèmes de la temporelle n'y occupent que quelques pages (les pages 98 à 101). Ces pages contiennent surtout des listes d'exemples, sans commentaires (mentionnons que ces listes sont loin d'être complètes). Une autre étude, plus récente, de Helga Kalén, Studia in Iordanem philologica (Uppsala, 1939) n'aborde aucunement les particularités des subordonnées temporelles.

10 Voir, à ce sujet, R. Iordache, "Cum" temporal o "cum" explicativo?, o Sobre la procedencia y los principales valores de la conjunción "cum", dans "Helmantica", XXX, Salamanque, 1979, pp. 264-65; ibid., p. 266.

11 Voir, l’étude citée dans la note 10, pp. 238-245 et pp. 270-276. 
cum historicum (plus rarement appelé cum descriptif; toujours un dérivé du cum instrumental-modal ${ }^{12}$ ), construit, généralement, à l'époque classique, avec le subjonctif, $u t$ et $u b i$.

A) Quand l'action de la principale coïncidait totalement comme durée avec l'action de la temporelle et que l'on insistait sur l'idée de durée égale, les conjonctions avaient le sens de "aussi longtemps que". Dans ce cas, les deux actions - celle de la subordonnée aussi bien que celle de la principale - sont duratives.

B) L'action de la principale pouvait avoir lieu:

1. dans un seul moment compris dans la durée de l'action ou de l'événement de la proposition temporelle, ou bien:

2. pendant plusieurs moments compris dans la durée de l'action de la subordonnée; enfin:

3. elle pouvait coïncider comme durée avec l'action de la temporelle sans qu'on insiste pour autant sur l'idée de durées égales, mais uniquement sur l'idée de simultanéité.

Dans toutes les situations, l'action dans la temporelle est durative.

Dans de telles propositions, les conjonctions temporelles avaient le sens de: "comme", "lorsque", "quand", "dans la période où", "pendant que".

Parfois - d'ailleurs assez rarement - la subordonnée temporelle du latin cicéronien indiquait une simple coïncidence temporelle. Mais, dans la plupart des exemples, il s'agit d'un rapport plus complexe, modal-temporal (ou même: locatif-modal- temporel). A la valeur modale-temporelle s'ajoutait souvent une nuance de cause, de condition, d'opposition concessive etc.

Généralement, dans le latin classique, cum narratiuum est suivi du subjonctif. Il s'agit d'un subjonctif "de caractérisation", ou "qualificatif"13, indiquant les conditions spéciales dans lesquelles se réalisait l'action de la principale. ${ }^{14}$

12 Voir l'étude citée dans la note 10, pp. 246-249.

13 Pour ce qui est du subjonctif de caractérisation, provenant du subjonctif potentiel, plus rarement volitif, dans les propositions relatives, à l'époque préclassique, voir R. Iordache, Relatives Causales ou Relatives Consécutives?, dans "Helmatica", XXVIII, Salamanque, 1977, p. 261, pp. 263-65, 269-73. Voir également R. Iordache, "Cum” temporalo "cum" explicativo?, op.cit., pp. $248-49$.

14 Au dire d'Otto Riemann, le subjonctif apparaît pour "caractériser la situation" (voir Syntaxe latine d'après les principes de la grammaire historique, Paris, 1935, $7^{\circledR}$ éd.. p. 422, par. 219, c); d'après Ch. E. Bennett, le subjonctif indique "une qualité du temps": "quom took a clause stating a quality of a time..." (pour le sens "quand", "lorsque" et "pendant que" de quom, voir Syntax of early Latin, Boston, Allyn-Bacon, 1910, vol. I, p. 303). M. Bassols de Climent et d'autres encore ont tort d'insister sur la valeur consécutive et non pas qualificative du subjonctif, dans les propositions introduites par cum narratiuum. Voici ce qu'en dit M. Bassols de Climent: "En un principio tenían estas oraciones un valor consecutivo muy acusado..." (Sintaxis latina, Madrid. 1976, $5^{e}$ éd., p. 330, par. 322). 
Les autres conjonctions se construisaient d'habitude avec l'indicatif. Cependant, le subjonctif qualificatif n'est pas exclu, notamment lorsqu'il s'agit de cum déterminatif (ou cum "uere temporale").

C) Il y avait aussi des situations contraires où l'action de la temporelle était momentanée et s'encadrait dans un seul moment de la durée de l'action de la principale; il se pouvait aussi que les deux verbes (de la temporelle et de la principale) présentassent des actions momentanées. Dans ces cas, les conjonctions avaient le sens de: "comme", "quand", ou "au moment où". Le rôle de ces propositions était d'indiquer d'une manière plus ou moins exacte le moment où s'était passée, se passait ou allait se passer l'action ou l'événement de la proposition corrélative.

A) Au sens de "tant que", "aussi longtemps que", on employait les conjonctions suivantes:

1. dum - construit souvent avec le véritable présent de l'indicatif et le présent historique; suivent, par ordre décroissant de la fréquence d'apparition dans les textes: l'indicatif parfait, imparfait et futur ${ }^{15}$; jamais, en latin cicéronien, le plus-que-parfait de l'indicatif et le futur antérieur;

2. quoad - surtout avec le parfait et le présent de l'indicatif;

3. quamdiu - construit avec l'indicatif présent, parfait etc.;

4. donec - fréquent à l'époque d'Auguste et construit pendant cette période avec le présent, l'imparfait, le parfait et le futur.

B) Au sens de “comme”, "pendant que", le latin cultivé à l'époque classique employait la conjonction dum, construite en général avec le présent historique, plus rarement avec le véritable présent de l'indicatif ${ }^{16}$ et encore plus rarement avec d'autres temps de l'indicatif: parfait, imparfait, futur.

Au sens modal-qualificatif-temporel de: "comme", "lorsque", "pendant que", cum narratiuum suivi de l'imparfait du subjonctif était de règle.

Pour le sens de: "comme", "quand", "pendant que", on utilisait cum "temporel proprement dit" ${ }^{\text {"17 }}$, construit d' habitude avec l'imparfait de l'indicatif, parfois avec le

15 Les temps de l'indicatif sont énumérés d'habitude par ordre de leur fréquence après la conjonction en cause. La fréquence d'apparition des divers temps de l'indicatif après chaque conjonction a été établie suivant les exemples et les indications du Thesaurus linguae Latinae et des principaux dictionnaires et grammaires du latin.

16 "le véritable présent (de l'indicatif)" - dans l'acception que donnent à cette forme R. Kühner et C. Stegmann, Ausführliche Grammatik der lateinischen Sprache, Hannover, 1971, 2. Teil, 1. Bd., p. $114, a$ et $c$.

17 appelé aussi "(relatif) temporel" ou "cum simplement temporel" - voir Al. Ernout - Fr. Thomas, Syntaxe latine, Paris, 1964, $3^{\mathrm{e}}$ éd.., p. 363, par. 359; ibid., p. 365, par. 361. 
présent réel, le présent itératif ou pro futuro, le futur, de même que le perfectum praesens; rarement apparaissait le présent historique ${ }^{18}$.

Le sens de "comme", "quand" pouvait aussi être rendu par ut et ubi construites avec l'imparfait de l'indicatif. Dans ce cas, à l'idée temporelle - exprimée par les propositions de ce genre - s'ajoutait d'habitude une nuance causale. Vt et ubi apparaissent aussi avec le présent historique, le présent réel et le futur.

En latin vulgaire, on utilisait au sens de "quand" quando. En corrélation avec certains adverbes (tum, tunc etc.) ou locutions adverbiales (eo tempore, postero die etc.), quando pouvait désigner un rapport temporel précis entre l'action ou l'événement de la principale et l'action ou l'événement de la subordonnée.

C) Au sens de "quand", "au moment où", “juste au moment où", on utilisait cum "temporel proprement dit", construit avec le présent réel, le présent itératif et le présent-futur, ainsi qu'avec le futur antérieur et le parfait historique. Il était rarement suivi du présent historique et du futur.

Pour les actions momentanées, on pouvait employer aussi $u t$, de même que $u b i$, le plus souvent avec le parfait historique et, plus rarement, avec le présent historique, le présent itératif et le futur (pour des actions répétées ou qui ne se produisent qu'une seule fois).

En latin familier et vulgaire, cum trouve un équivalent plus ou moins exact en quando.

Dans les oeuvres de Jordanès, les propositions temporelles de simultanéité sont introduites par les conjonctions suivantes, que nous énumérons par ordre décroissant de leur fréquence d'apparition dans les textes: dum, cum, quando, ut, ubi, donec, quamdiu, quousque et quod (sans compter les exemples de conjonctions itératives).

Parmi ces conjonctions, dum est de loin la plus fréquemment utilisée pour indiquer le rapport de simultanéité. Le choix de dum comme la modalité la plus fréquente d'introduire des subordonnées temporelles de simultanéité (de divers types), de même que des temporelles de postériorité, ainsi que d'exprimer des relations de subordination causale et adversative-concessive, s'explique tant par la tendance à remplacer, en latin vulgaire ${ }^{19}$, la conjonction cum, surchargée de valeurs et manquant d'expressivité et précision, que par la prédilection de l'auteur pour la conjonction dum.

18 voir note 15 .

19 Dum, beaucoup employé en bas latin, s'est transmis dans les langues romanes (à l'exception du roumain), soit sous la forme dunc $>$ it. dunque, fr. donc, soit uni aux adverbes interim ou interea, voir ancien port.: dementre, anc. esp.: demientre, ancien français, ancien portugais: (en)dementres, dementre que et domentre que (voir Wilh. Meyer-Lübke, RomanischesEtymologisches Wörterbuch, Heidelberg, 1935, $3^{\mathrm{e}}$ éd., $\mathrm{n}^{\circ}$ 2794). A noter que dum a donné naissance soit à des particules conclusives, soit à des adverbes et conjonctions temporelles, soit enfin à des conjonctions causales et adversatives-concessives (dans les derniers cas, en se combinant avec la conjonction "quod"). 
A) Les conjonctions qui, chez Jordanès, indiquent que l'action de la proposition principale se déroule pendant une période de temps égale à celle de l'action de la subordonnée sont: dum, donec, quamdiu et quousque.

Dum au sens de "aussi longtemps que" est rare chez Jordanès en contraste avec le grand nombre d'exemples de dum au sens "pendant que" - sens que la disparition en bas latin de cum narratif aura probablement renforcé. Bien sûr, on pourrait donner une autre explication aussi: c'est que les auteurs tardifs préfèrent à dum (au sens de "aussi longtemps que") d'autres adverbes ou locutions adverbiales comme quamdiu, quantum, in quantum, in tantum quod, in tantum, qua, in quo etc.

Dum au sens "aussi longtemps que" apparaît uniquement avec l'imparfait du subjonctif -5 exemples. D'autre part, il existe plusieurs exemples de dum explicatif à évidente nuance causale, suivi de l'indicatif et du subjonctif (la nuance causale est dérivée du sens "pendant que", et du sens "aussi longtemps que").

Le subjonctif précédé par dum (= "aussi longtemps que") apparaît dès Plaute (voir Truc., 234) et Térence (Heaut., 1058) et il est assez fréquent à toutes les époques (voir Varron, R. r., 1, 1, 3; Ciceron, Verr., 2, 2, 50; Tite-Live, 7, 37, 10; Velleius Paterculus, 1, 2, 3; Pline, Nat. hist., 14, 146; Suétone, Aug., 78, 2 etc. etc.) - il s'agit, évidemement, du subjonctif imparfait et - plus rarement - présent, marquant, tous les deux, la simultanéité. Après dum (= "aussi longtemps que"), le parfait et le plus-queparfait du subjonctif sont extrêmement rares: le parfait est attesté chez Cicéron, Verr., 2, 3, 224; ibid., 2, 1, 12 et Tacite, Ann., 11, 28; le plus-que-parfait apparaît chez Quintilien, Inst. or., 6, 3, 51 et Pseudo Frontinus, Strat., 4, 1, 44. 20

Chez Plaute et Térence, tout comme chez les écrivains classiques où les écrivains qui - dans les siècles suivants - imitent le modèle classique, l'emploi du subjonctif était justifié par la présence d'une nuance de répétition, du style indirect, de l'attraction modale, une nuance d'irréalité etc. Chez les auteurs qui subissent l'influence du latin vulgaire et particulièrement dans le latin des $\mathrm{IV}^{\mathrm{e}}-\mathrm{VI}^{\mathrm{e}}$ siècles (Ammien etc.), le subjonctif qui accompagne dum explicatif-temporel (avec le sens "aussi longtemps que") n'est pas motivé par rapport à la norme classique.

L'apparition du subjonctif dans les subordonnées de ce type, à l'encontre de l'usage classique, pourrait s'expliquer par la confusion existant en latin parlé et surtout en latin vulgaire à l'égard de l'emploi des modes, confusion qui s'accentue après la chute de l'Empire Romain d'Occident. Les gens du peuple qui connaissaient vaguement ou ignoraient les normes du "latin d'or", utilisaient souvent l'indicatif à la place du subjonctif et vice versa. Ceci a eu pour conséquences l'affaiblissement de la valeur modale de l'indicatif et, à plus forte raison, du subjonctif, et l'utilisation de ces modes d'une manière étrangère à l'usage classique, même par des écrivains qui prétendaient être de bons connaisseurs du latin classique. ${ }^{21}$.

20 Voir Thesaurus linguae latinae, vol. V, pars 1, Leipzig, 1934, pp. 2222-23.

21 A propos de cette question voir aussi $\mathrm{H}$. Goelzer, Etude lexicographique et grammaticale de la latinité de Saint Jérôme, Paris, 1884, p. 362: "En résumé, l’indicatif et le subjonctif sont employés dans Saint 
A la manière de bien d'écrivains tardifs, Jordanès emploie fréquemment et pour des raisons plus ou moins spéciales et évidentes l'indicatif à la place du subjonctif (dans l'interrogative indirecte, la relative qualificative à nuance causale, la subordonnée consécutive, la concessive introduite par cum, quamuis, licet, dans la subordonnée causale introduite par quia - subordonnée qui se trouve dans une étroite dépendance subjective vis-à-vis de la régissante etc.) et le subjonctif à la place de l'indicatif (dans la relative-adjective dépourvue de nuance qualificative, dans la causale introduite par quod et quia exprimant une cause réelle etc).

Une explication fort plausible de l'apparition du subjonctif après dum (= "aussi longtemps que") serait l'influence de la construction de cum narratif avec le subjonctif et même de la construction avec le subjonctif de dum ayant le sens "pendant que" (voir le commentaire dans les pages suivantes).

Comme nous l'avons déjà précisé, chez Jordanès, dum explicatif-temporel au sens de "aussi longtemps que" est construit uniquement avec l'imparfait du subjonctif. Aucun des cinq exemples ne présente de corrélatif; voici l'exemple des Getica, 296: "...numquamque Gothus Francis cessit, dum uiueret Theodoricus."

Remarquons que, dans deux cas, dum forme une locution à côté de l'adverbe usque $^{22}$. Voir Getica, 100: “...suaque in patria feliciter in pace uersantur, usque dum eorum praeuius existeret ${ }^{23}$ Ostrogotha."; ibid., 303: “...et usque dum uiueret (Thiudis), Vesegothas contenuit. ${ }^{24 "}$. Les locutions conjonctives apparaissent néanmoins très fréquemment par rapport au nombre total des exemples où dum a ce sens. L'adverbe usque était nécessaire pour établir une distinction entre $d u m$ au sens de "aussi longtemps que" et dum au sens de "pendant que".

Nous mentionnons que les verbes des subordonnées en discussion sont: esse, exsistere (en tant que synonyme de esse), uiuere ou son composé aduiuere ${ }^{25}$ (comportant le sens de uiuere), donc des verbes exprimant des actions duratives. C'est pourtant, rappelons-le, le parfait du subjonctif qui aurait dû être employé.

On constate que tous ces exemples, construits avec l'imparfait du subjonctif, apparaissent dans les Getica, dans des passages faisant l'éloge des Goths ou de certains rois des Goths. En de telles situations, l'emploi du subjonctif imparfait nous paraît être une preuve des efforts que fait l'auteur pour s'exprimer d'une manière élégante, précieuse (voir en plus la présence de la locution usque dum).

Jérôme, comme dans la latinité postérieure, d'une façon beaucoup plus libre." (C'est nous qui soulignons les derniers mots du passage cité).

22 Vsque est, d'ailleurs, un adverbe assez fréquent chez Jordanès: il forme des locutions prépositionnelles avec ad et in; seul, suivi de l'accusatif, il a valeur de préposition; il accompagne divers adverbes: hactenus, nunc; il précède dum avec le sens "aussi longtemps que" ou "jusqu'à ce que".

23 pour exsisteret.

24 contenuit, pour continuit (voir aussi des formes telles que obtenuit, subegeret, en échange, voir autre type d'erreurs: subigisset etc.)

25 composé rarement employé et uniquement en bas latin. 
En ce qui concerne la concordance des temps, les règles sont observées. D'habitude, dans la principale on retrouve le parfait de l'indicatif (voir Getica 246, 296, 303), une seule fois - le présent historique (Get., 100) et, une autre fois, l'imparfait du subjonctif (dans le cadre d'une relative consécutive remplissant le rôle de régissante Get., 303).

Les autres conjonctions qui apparaissent chez Jordanès avec le sens "aussi longtemps que" sont: donec, quamdiu et quousque. Quamdiu (conjonction spécifique du sens “aussi longtemps que") et quousque n'apparaissent qu'une seule fois chez Jordanès et avec ce seul sens; donec est attesté une seule fois avec le sens de "aussi longtemps que" et deux fois avec le sens: "jusqu'à ce que" (voir ci-dessous le paragraphe "la relation temporelle de postériorité").

Un fait intéressant est l'apparition de quousque en tant que conjonction, comportant le sens "aussi longtemps que"26. Le choix de ce composé est dû, en grande partie, à l'adverbe usque - fréquemment utilisé à l'époque tardive (voir, par exemple, la formation "quoad usque" chez Cassien, Con., 12, 6, 9; voir aussi "usquequo" dans Vitae Patrum, 3, 61).

L'emploi rare des conjonctions donec et quamdiu, ainsi que l'absence de quoad chez Jordanès correspond à une tendance générale à éviter, en bas latin, ces conjonctions, surtout donec et quoad ${ }^{27}$, situation que confirme l'inexistence de donec et quoád dans les langues romanes (quamdiu est partiellement transmis ${ }^{28}$ ). Mais, puisque quousque - conjonction de date assez récente - apparaît, lui-aussi, une seule fois, force nous est de penser à une autre explication de ce phénomène: c'est que Jordanès n'aura pas eu intérêt à construire des subordonnées de ce type.

Dans la série de conjonctions mentionnées, seul donec est construit avec l'indicatif suivant les règles classiques, voir Romana, 107: "Tamdiu superbiam regis populus Romanus perpessus est, donec aberat libido." - passage copié, d'ailleurs, sur Florus, Epit., 1, 1, 10.

Les autres conjonctions sont suivies du subjonctif, mais ce subjonctif ne saurait être motivé ni par le style indirect, ni par l'attraction modale:

1. "Quod praeceptum quamdiu Athalaricus res eiusque mater aduiuerent, in omnibus custodientes pene ${ }^{29}$ per octo annos in pace regnarunt.", Get., 305;

2. "Cuius Candacis Alanouiiamuthis ${ }^{30}$ patris mei genitor Paria, id est meus auus, notarius; quousque Candac ${ }^{31}$ ipse uiueret, fuit (notarius)...", Get., 266.

26 Dans sa dissertation, Fr. Werner (op.cit.) ne remarque pas la présence de la conjonction quousque dans les Getica.

27 Voir Fried. Stolz -J.G. Schmalz, Lateinische Grammatik, München, 1928, $5^{\mathrm{e}}$ éd., 2. Teil, 2. Bd., pp. 754 et 769; voir aussi J. B. Hofmann - A. Szantyr, München 1965, 2. Teil, 2. Bd., pp. 606, 629, 654.

28 Il s'est conservé seulement en provençal, sous la forme quandius - voir Wilh. Meyer-Lübke, $R$. $E$. W., op.cit., 6929.

29 La graphie pene, fréquente chez Jordanès, reproduit l'usage courant depuis longtemps dans la prononciation vulgaire. 
Rappelons que le premier fragment concerne le roi des Ostrogoths, Athalaric, et sa mère, Amalasuentha. Le second fragment concerne le grand-père de notre auteur.

Vu le contenu de ces phrases, nous sommes d'avis que le subjonctif imparfait appartiendrait, ici, au style emphatique, comme dans le cas des passages cités antérieurement (contenant la conjonction dum). A noter que les deux passages se trouvent dans les Getica, ouvrage dont on peut dire qu'il indique, dans une plus grande mesure que les Romana, un style propre à l'auteur que nous étudions. L'imparfait utilisé dans ces exemples (à la place du parfait) reflète l'influence de la manière de construire cum narratif.

Dans les deux exemples, la concordance des temps est réalisée en accord avec l'usage classique.

On constate que certaines de ces conjonctions ne sont pas accompagnées d'adverbes et n'ont pas de corrélatifs dans la principale.

Précisons que les antécédents n'apparaissent que dans des contextes où l'on trouve des conjonctions anciennes et à plusieurs valeurs et ayant, parfois, un corps phonétique très réduit. Ainsi, seul dum (au sens de "aussi longtemps que") forme une locution conjonctive avec l'adverbe usque (voir les exemples cités à la page 39); l'emploi de l'adverbe usque à côté de la conjonction dum est très fréquent. Donec a pour corrélatif l'adverbe tamdiu. Les conjonctions récentes, plus vigoureuses, n'avaient pas besoin de pareilles précisions, par exemple, la conjonction quousque. Quamdiu, conjonction dont le sens est évident et qui présente un volume phonétique satisfaisant, n'a besoin d'aucun antécédent.

Ajoutons que les quelques antécédents qui apparaissent dans le cadre de ce type de temporelle - usque et tamdiu - sont fréquents à l'époque tardive.

B) Dans le cadre du rapport temporel de simultanéité, dum apparaît très souvent avec le sens de "pendant que".

Avec cette valeur, dum est suivi:

I. 1. de l'indicatif présent, en fait le présent historique, auquel correspond dans la principale:

a) le présent historique (Jordanès manifeste en général une préférence très marquée pour l'emploi du présent historique) - voir Romana, par. 241; 350, 378, 380 etc., Getica, par. 129, 220, 298 etc., ou: b) le parfait de l'indicatif. voir Romana, par. 6, 51, 213, 305, 346, 373 etc., Getica, par. 235, 247, 312 etc.;

2. de l'indicatif parfait (beaucoup moins souvent), tandis que le verbe de la principale se trouve toujours à l'indicatif parfait - voir Romana, 277 et 349. L'origine expli-

30 A propos de ce nom propre, voir Th. Mommsen, dans sa préface à l'édition citée, p. 6.

31 Candac pour Candax? (tout comme les formes fautives qui apparaissent chez Jordanès: Xerses, ou senes et senis etc.). Voir cependant Ellac ("Attilae filius") - Getica, 262. 
cative, instrumentale-modale de dum est particulièrement évidente dans les exemples avec l'indicatif parfait; “... dum (Caracalla) contra Persas mouit procinctum (exercitum), Osroene ${ }^{32}$ Edessa ${ }^{33}$ defunctus est.”, Rom., 277 (L'idée est reprise du Breuiarium de uictoriis et prouinciis populi Romani 21, de Sex. Rufus Festus: "expeditionem in Persas parans, in Osroena apud Edessam propria morte obiit.") Dans l'exemple cité, dum a le sens de "par le fait que" $\rightarrow$ "pendant que".

3. de l'indicatif imparfait (un seul exemple de ce genre), suivi dans la principale de l'indicatif parfait - voir Romana, 279: "Sed imperator, dum nullum genus obscenitatis in regno suo quod non faceret praetermittebat, occisus est tumultu militari." A noter la nuance causale qui se fait sentir dans cet exemple. La norme classique est respectée en ce qui concerne la fréquence d'apparition des divers temps de l'indicatif dans la proposition temporelle introduite par dum au sens de "pendant que": le plus souvent, c'est le présent de l'indicatif qui est employé, le parfait n'apparaissant que rarement ${ }^{34}$ (Cfr. la manière d'utiliser les temps dans les propositions introduites par cum identicum ou cum coincidens ${ }^{35}$ ). L'imparfait de l'indicatif - qui représente un écart par rapport aux règles classiques - n'apparaît qu'une seule fois.

L'observation de la soi-disante "règle du présent" aura été, dans une certaine mesure, une conséquence de la prédilection de l'auteur pour l'emploi du présent historique - temps utilisé non seulement dans les subordonnées de divers types, mais encore dans les propositions corrélatives respectives, de même que dans les propositions principales de différentes phrases. ${ }^{36}$

On peut cependant affirmer que la "règle du présent" de dum (au sens de "pendant que") était connue à Jordanès qui l'appliqua dans ses deux ouvrages non seulement dans les subordonnées explicatives-temporelles, particulièrement nombreuses, mais encore dans les propositions adversatives- concessives commençant par dum (voir Getica, 227 et 312) et, surtout, dans les subordonnées explicatives-causales qu'introduit $d u m$, au sens de "pendant que" $\rightarrow$ "puisque", "du moment où". ${ }^{37}$ En ce

32 Nom de région sans la préposition in (moins vraisemblablement conçu comme adjectif-ablatif déterminant le nom Edessa).

33 Ablatif, à la place du Locatif (Cfr. Rufus Festus, 21).

34 Pour ce qui est de la fréquence des différents temps de l'indicatif après dum = "pendant que", chez les auteurs classiques, voir Thesaurus linguae Latinae, vol. V-1, op.cit., pp. 2203-2207 (l'article sur dum); voir aussi Al. Emout - Fr. Thomas, Syntaxe latine, op.cit., p. 371, et d'autres grammaires encore.

35 Au sujet de cette question, voir R. Iordache, "Cum" temporal o "cum" explicativo?, op.cit., pp. 238-43; pp. 245-49.

36 A propos de l'emploi du présent historique dans les propositions principales, voir Fr. Werner, op.cit., p. 88. Voir également R. Iordache, Observaciones sobre la subordinada causal en las obras de Jordanes, concernant la fréquence du présent historique dans les subordonnées explicatives-causales introduites par dum provenant du sens “aussi longtemps que”, dans les principales régissant les propositions introduites par ut etc. (op.cit., p. 11, pp. 13-14, p. 31, p. 36, p. 41, p. 45 etc.)

37 Voir R. Iordache, Observaciones sobre la subordinanda causal en las obras de Jordanes, op.cit., p. 31, pp. 39-41. 
qui concerne l'observation de la "règle du présent", voir, dans les pages suivantes, le commentaire au sujet de l'emploi du présent historique dans les subordonnées introduites, chez Jordanès, par cum, ubi et $u t$, temporels et explicatifs-causals. ${ }^{38}$

Les temporelles introduites par dum (= "pendant que") et construites avec l'indicatif n'ont pas de corrélatifs dans la principale.

\section{Dum au sens de "comme", "pendant que", est souvent suivi du subjonctif.}

L'apparition du subjonctif après dum (il s'agit en général du subjonctif imparfait et, plus rarement tout le long de la latinité, du subjonctif présent), dans des situations où sa présence ne saurait être expliquée par une nuance finale ou potentielle, par le style indirect, par une attraction modale etc. etc. est un phénomène spécifique du latin parlé, dû à l'analogie avec cum narratiuum (ou historicum) exprimant la simultanéité. Les premiers exemples indubitables du subjonctif imparfait, à l'encontre des normes classiques, apparaissent dans le Bellum Africum, 25, 1 et le Bellum Hispaniense, 23, 2; en poésie, les premiers exemples sont de Virgile, Georg., 4, 457; Aen., 10, $799^{39}$. Les premiers exemples certains du subjonctif présent sont attestés chez Lucrèce, $R$. n.., 1, 499, ensuite dans Lydia, v. 19 (Cfr. Plaute, Pseud., 629).

Très répandu en bas latin ${ }^{40}$, le subjonctif imparfait précédé de dum au sens de "pendant que" est fréquent chez Jordanès aussi, voir Romana, 226: "Se ${ }^{41}$ quoque dum de inimicis ${ }^{42}$ ulcisceretur, defunctus Romanos per testamentum heredes reliquid." ${ }^{43}$; ibid., par. 234: "Pompeius autem maioris Armeniae regem dum persequeretur... ...ille in Artaxata urbe ... diademam suam Pompeio optulit." ${ }^{44}$ Voir également Romana, 217, 294, 341, 369 etc.; Getica, 27, 136, 157, 223 etc.

38 Pour l'existence - dès la période préclassique - de la "règle du présent" dans le cas de nombreuses conjonctions temporelles et explicatives-causales, telles que cum, quoniam, dum, ubi, ut etc., voir $\mathrm{R}$. Iordache, "Cum" temporalo "cum" explicativo?, op.cit., pp. 260-61.

39 D'autres exemples aux I $\mathrm{I}^{\mathrm{er}}$ et $\mathrm{II}^{\mathrm{e}}$ siècles de notre ère chez: Hygin, Fab., 12 $(43,19), 18(51,12)$; Valère Maxime, 3, 3, 2; 9, 12, 7; Phèdre, 1, 4, 2; Valérius Flaccus, 1, 425; Justin, 9, 6, 3 et d'autres. Le subjonctif imparfait après dum (="pendant que") est cependant absent chez Tacite, Florus et même Apulée; les auteurs tardifs l'emploient pourtant de plus en plus souvent (voir la note suivante).

40 Voir Aurélius Victor, Caes., 39, 12; Vir ill., 35, 10; Jérôme, Ep., 10, 3; 22, 30; 48, 18; 52, 1; 60, 5; 125, 12;130, 16; V, Pauli, 5; V.Malch., 2; Sulpice-Sévère, Martin., 8, 12;12, 1; Claudien, Gigantom., 101; In Eutrop., 2, 413; Cassien, Con., 12, 6, 4; ibid., 15, 10, 1; ibid., 17, 5, 1 etc.; Sidoine Apollinaire, Ep., 4, 11; Victor de Vite, Persec. Vand., 2, p. 14; Avit, p. 64, 11; 112, 6 etc.; Boèce, Consol. phil., 1, 1; Fulgence, Myth., 2, 9 etc.; Grégoire de Tours, Hist. Franc., 2, 8; 4, 3 etc. etc. Pour d'autres exemples, voir Thesaurus linguae Latinae, vol. V-1, op.cit., p. 2219, 1. 53 sqq. Voir aussi J. B. Hofmann - A. Szantyr, Lateinische Grammatik, 2. T., 2. Bd., op.cit., p. 614, et d'autres grammaires.

41 se ulcisceretur, à la place de ulcisceretur (la même erreur dans les Getica, 275).

42 Ablatif prépositionnel au lieu de l'accusatif non prépositionnel (Cfr. franç.: "se venger de quelqu'un"; esp.: "vengarse de uno" etc.).

43 reliquid pour reliquit apparaît dans d'autres passages aussi (voir Romana, 54, 223 etc.). Il y a, chez Jordanès, $d$ 'autres formes également fautives, dues à la confusion entre $d$ et $t$, par exemple: ad (pour at) et vice versa, haut, inquid, pugnad etc. La confusion des consonnes $d$ et $t$ est relativement fréquente dans les Romana et les Getica. optulit - l'assimilation de la consonne $b$ du préfixe $o b$ - est assez fréquente chez Jordanès (voir aussi 
Les exemples des verbes au subjonctif imparfait sont plus nombreux dans les Getica que dans les Romana, mais cette différence n'est pas grande: dans les Romana, on retrouve neuf exemples de la conjonction dum suivie de neuf verbes; dans les Getica, la conjonction dum apparaît neuf fois également, mais les verbes qu'elle précède sont au nombre de douze - sans compter les situations où dum a un évident caractère causal.

Si les subordonnées sont construites avec l'imparfait du subjonctif, le verbe de la proposition principale est, en général, à l'indicatif parfait (voir ci-dessus Romana, 226 et 234; voir aussi Romana, 217, 294; Getica, par. 27, 305 etc.). Au contraire, si le verbe des propositions introduites par dum (= "pendant que") est à l'indicatif, le présent historique est le plus souvent d'usage dans les principales. Enfin, dans la plupart des cas chez Jordanès, cum narratif de simultanéité a pour corrélative une proposition dont le verbe est au parfait de l'indicatif.

On pourrait, certes, se demander si le subjonctif utilisé après dum n'a pas, toutefois, une valeur qualificative, du moins dans certains cas. En voici un exemple tiré des Getica, 83: "... dum in Christianos arma commoueret, imperium simul et uitam amisit." Voir aussi les exemples cités ci-dessus.

Le subjonctif qualificatif était sans doute familier à Jordanès qui l'utilise dans un grand nombre de subordonnées, à savoir: des relatives consécutives, des relatives à nuance causale et concessive, des subordonnées introduites par cum narratiuum etc. D'autre part, on constate qu'il y a peu d'exemples de cum narratiuum suivi du subjonctif et que, en outre, la subordonnée "narrative" (à valeur explicative-qualificative-temporelle) introduite par cum est parfois construite, chez Jordanès, avec l'indicatif. Malgré ces faits qui sembleraient infirmer la thèse de l'utilisation, chez Jordanès, de dum "subjonctive" à valeur qualificative, notre auteur comprend parfois et emploie correctement la valeur du subjonctif dans les propositions narratives qui commencent par dum et, naturellement, par cum.

D'ailleurs, fortuitement ou non, la manière même dont Jordanès utilise d'une manière constante le parfait de l'indicatif dans les principales des propositions introduites par dum suivi du subjonctif est proche de la façon dont sont construites les principales de propositions de cum narratiuum suivi du même subjonctif; on en dirait une tentative de différencier dum narratif (suivi du subjonctif) et dum construit avec l'indicatif.

Un aspect particulièrement intéressant est l'apparition, dans la même phrase, de dum (= "pendant que") suivi de l'indicatif et du subjonctif à la fois, voir Getica, 93: “... Marciae sororis suae puella, dum lauat ${ }^{45}$ in flumine illo ... exindeque uellit ${ }^{46}$

Romana, 366: optulit). Pourtant les Getica, 112 donnent obtulere. Dans le cas d'autres préfixes, on rencontre la situation inverse: le préfixe est restitué dans sa forme originelle, même si, dans les composés, sa dernière consonne se trouvait couramment assimilée; cela prouve la préciosité qui apparaît parfois dans la graphie et, en général, dans la manière de s'exprimer de Jordanès. 
aquam haurire, casu uas aureum quod ferebat in profundum decidit ... longeque post ab imis emersit."

Voici la traduction du fragment: "Pendant que la fille de sa soeur, Marcia, se lavait dans cette rivière ... et que (comme, du moment où) elle voulait y puiser de l'eau, un vase en or qu'elle tenait dans la main coula par hassard au fond de la rivière ... et, longtemps après, remonta à la surface."

Bien qu'encadré uniquement par des verbes à l'indicatif (car la phrase, beaucoup plus ample que le passage cité, ne comporte que des verbes à l'indicatif), uelle se trouve au subjonctif.

On se demande alors: s'agit-t-il, dans ce cas, d'une association involontaire des aspects classiques et vulgaires, ou bien Jordanès aura-t-il voulu exprimer, par l'intermédiaire de uellit (en fait, uellet - voir note 46), un type spécial de rapport entre la subordonnée et la principale (rapport que le latin classique rend par cum narratiuum, ou cum narratif-causal)?

Evidemment, la réponse à cette question n'est pas facile. L'étude de la concordance des temps dans cette phrase ne nous avance guère, car l'emploi du subjonctif imparfait correspond aux normes classiques.

Rappelons toutefois que des situations où dum est suivi de deux modes apparaissent chez d'autres auteurs aussi: voir, à l'époque classique, Varron, $R$. r., 1, 2, 12 (dum au sens de “jusqu'à ce que"). D'autre part, il existe, dans les oeuvres de Jordanès, des phrases où la même conjonction dum introduit simultanément des subordonnées à nuances différentes: l'une à valeur explicative-concessive, l'autre à valeur explicative-causale, avec, dans les deux cas, le subjonctif imparfait (voir Getica, 290). Mais il se peut aussi que la conjonction et le mode diffèrent, bien que les propositions soient proches ou même identiques au point de vue de leur valeur sémantique - voir Roma$n a, 305$, où cum narratif suivi du subjonctif alterne avec dum explicatif-temporel construit avec l'indicatif (nous renvoyons au commentaire concernant cum narratiuum). Ajoutons également la réunion du subjonctif et de l'indicatif dans les subordonnées consécutives, à l'intérieur de la même phrase (voir Romana, 265; Getica, 278),

Très rarement, dum est suivi du présent du subjonctif, voir Getica, 29: "in quam sententiam $^{47}$ et nonnulli consensere maiorum: Ioseppus ${ }^{48}$ quoque annalium relator uerissimus dum ubique ueritatis conseruet regulam et origines causarum a principio

46 uellit pour uellet. A propos de la confusion des voyelles e et $i$ dans divers mots (noms, adjectifs, verbes etc.) et dans diverses positions (syllabes finales, initiales ou média nes), voir aussi les notes suivantes. Dans le cas de uellit, la confusion a été favorisée par la ressemblance de forme entre le subjonctif présent et imparfait du verbe uelle. Vellit pour uellet apparaît encore une fois chez Jordanès, à savoir dans les Getica, 283.

47 Complément à l'accusatif, à la place de l'ablatif prépositionnel (avec in ou, surtout, de).

48 Ioseppus pour Iosephus. On rencontre aussi, chez Jordanès, le phénomène contraire: $p h$ pour $p p$ ou $p$, voir Sardanaphalus, même Sardanafalus (Rom., 49). 
reuoluat." Si l'utilisation du temps (présent) est correcte, les raisons de l'emploi du subjonctif ne sont pas claires: serait-ce à cause de l'idée de style indirect, de la nuance causale (subjective) ou bien de l'influence de cum narratif?

Un exemple semblable apparaît dans les Romana, 7 :

"Quod quamuis simpliciter reor ${ }^{49}$ dictum uideri ${ }^{50}$ doctissimis, gratum tamen fore aestimo mediocribus, dum et breuia sine fastidio legant et sine aliquo fuco uerborum quae lectitauerint sentiant."

Bien que dum suivi du subjonctif soit fréquemment attesté dans les Romana et les Getica, c'est pourtant la construction classique - à savoir dum suivi de l'indicatif présent - qui prédomine numériquement.

En même temps, précisons-le, dum suivi du subjonctif imparfait est plus fréquent que le cum historicum suivi de l'imparfait du même mode.

L'unique corrélatif qui existe pour dum suivi du subjonctif, c'est l'adverbe ilico (en fait, il n'apparaît que pour dum construit avec le subjonctif imparfait). Ilico, corrélatif fréquent dans les oeuvres des écrivains soumis à l'influence du latin vulgaire, est attesté, chez Jordanès, deux fois seulement et sous la forme correcte ilico (et non pas illico). Il est intéressant de remarquer que ces deux exemples font partie des Getica. Dans chacun d'eux, ilico est placé au début de la principale, voir Getica, 136: "Nam in alia parte socios eius reclausos ${ }^{51} \mathrm{dum}$ milites ducis sui iussu trucidare conarentur et uox morientium duriter emissa iam suspectis ${ }^{52}$ auribus intonaret, ilico aperto dolo cognoscens Fritigernus ${ }^{53}$ euaginato gladio e conuiuio non sine magna temeritate uelocitateque egreditur..."; voir également Getica, 102.

Une autre conjonction que Jordanès utilise afin d'indiquer la relation temporelle de simultanéité c'est cum narratif.

Par rapport à l'usage classique, cum narratif est rarement employé chez Jordanès. D'autre part, comme nous avons déjà affirmé, les subordonnées temporelles introduites par cum narratif construit avec le subjonctif imparfait - abstraction faite de cum narratif à caractère causal et de cum narratif concessif - sont, chez Jordanès, plus rares que les temporelles introduites par dum construit avec le subjonctif.

Pour ce qui est des subordonnées temporelles introduites par cum accompagné de l'imparfait du subjonctif, nous devons en distinguer deux catégories, dans les écrits de Jordanès et d'autres auteurs tardifs:

$49 \quad$ Remarquons la présence de l'indicatif dans la subordonnée introduite par quamuis.

50 Infinitif présent au lieu de l'infinitif futur ou de la périphrase "posse uideri". Pour d'autres exemples de ce type, voir $R$. Iordache, L'infinitif dans les oeuvres de Jordanès, dans "Linguistica", XXIV, Ljubljana 1984, pp. 144-46.

51 Cas intéressant de recomposition, à l'époque tardive, du composé qui avait subi la fermeture de la diphtongue du radical.

52 suspectis, au lieu de suspiciosis.

53 Fritigernus pour Fridigernus. La forme fautive est assez fréquente chez Jordanès. 
1. des subordonnées explicatives-temporelles où le subjonctif - employé selon les règles classiques - a une valeur qualificative;

2. des subordonnées temporelles, à caractère plus ou moins explicatif, où le subjonctif imparfait est, en fait, l'équivalent de l'indicatif imparfait.

Pour la première catégorie, voir Romana, 139: “... cum per Italiam naufragia sua latius traherent, sic persecuti sunt (Romani), ut..." (passage d'ailleurs copié sur Florus, 1, 13, 19). Voir également Getica, 196: "Cumque Attila necem Aetii .... uel cum sua ${ }^{54}$ perditione duceret expetendam .... circa nonam diei horam proelium sub trepidatione committit...".

Pour la seconde situation, voir Romana, 193: "Denique uix suffecere tabulae, uix scribarum manus..., cum priuatorum opes in publicum referrentur." (passage copié sur Florus, 2, 6, 25). A noter, dans la phrase qu'on vient de citer, la position de la subordonnée.

Parfois, la subordonnée introduite par cum est précédée d'expressions temporelles précises qui contribuent à appauvrir le contenu de la subordonnée. Voir Getica, 85: "Tertia ${ }^{55}$ post haec die, cum imperator prodiret ad campum, uidit eum exultantem 56 more barbarico iussitque tribuno, ut ${ }^{57}$..." (Cfr. Vita Maximini, 3: "tertia forte die cum processisset Seuerus ad campum, in turba exultantem more barbarico Maximinum uidit iussitque statim tribuno ut...".)

Mentionnons, dans un autre ordre d'idées, que parfois cum suivi de l'imparfait du subjonctif et $d u m$ construit avec l'indicatif alternent, dans la même phrase, pour des subordonnées rapprochées comme valeur. Voir Romana, 305: "Vnde egressus, dolo cuiusdam transfugae in deserta perductus, cum ui sitis, ardore solis confectus periret exercitus, ipse tantorum discriminum anxius, dum per uasta deserti incautius euagatur, ab obuio quodam hostium equite conto ilia ${ }^{58}$ percussus interiit...". D'ailleurs, le fragment est copié sur Orose, $A d u$. pagan., 7, 30.6. On se demande alors: pourquoi Jordanès n'a-t-il pas changé la manière de construire les temporelles copiées sur Orose? A ce qu'il paraît, pour des raisons stylistiques, afin d'éviter l'accumulation, dans la même phrase, des subordonnées introduites par la conjonction cum.

Voici une phrase tirée des Getica, 200, construite d'une manière similaire, bien qu'il soit impossible de supposer, dans ce passage, l'influence d'Orose ou d'autres historiens: "Ibique ${ }^{59}$ cum diu multumque ${ }^{60}$ obsidens nihil paenitus ${ }^{61}$ praeualeret, for-

54 Pour des raisons stylistiques, l'adjectif possessif est placé avant le nom qu'il détermine.

55 Dies est parfois au féminin chez Jordanès. Le latin vulgaire semble avoir préféré le féminin. Dies féminin apparaît chez d'autres auteurs tardifs aussi - voir Cassien, Inst., 1, 1, 4; ibid., 3, 4, 3; 4, 27, 3 etc.

56 pour exsultantem.

57 A propos de iubec suivi d'une complétive ayant le verbe au subjonctif, voir R. Iordache, $L$ 'infinitif dans les oeuvres de Jordanès, op.cit., p. 136.

58 Accusatif de relation, imitant la construction classique, affectée.

59 - que est fréquemment uni à des adverbes, ayant le rôle de réaliser le passage d'une phrase à autre. 
tissimis intrinsecus ${ }^{62}$ Romanorum militibus resistentibus, exercitu iam murmurante et discedere cupiente, Attila deambulans circa muros, dum, utrum solueret castra an adhuc remoraretur, deliberat, animaduertit candidas aues, id est ciconias, qui ${ }^{63}$...".

En ce qui concerne les écarts aux normes classiques, ils sont de types différents:

a) Dans un exemple, l'imparfait du subjonctif est remplacé, fautivement, par le présent du même mode: "Quippe cum patrii soli gliba ${ }^{64}$ nulla sit, sed statim hostile pomerium $^{65}$, mediusque (sous-entendu: populus Romanus) inter Latium adque ${ }^{66}$ Etruscos .... conlocatus omnibus portis in hostem incurreret .... per singulos (uicinos) itum est ....", Rom., 119. (Cfr. Florus, 1, 3, 7: "quippe cui patrii soli glaeba nulla, set statim hostile pomerium, mediusque inter Latium adque Etruscos .... conlocatus omnibus portis in hostem incurreret .... per singulos - uicinos - itum est...").

b) Parfois, le subjonctif imparfait de la subordonnée de cum narratif est substitué par l'indicatif présent: "Cumque his nouis nuptiis delectatus aulam regiam fouit ${ }^{67} \mathrm{Ra}$ uenna ${ }^{68}$, Roma egressus imperialis exercitus munita utriusque Tusciae loca inuadit.", Get., 311. La traduction de cette phrase est la suivante: "Et pendant que Witigès, heureux de ce nouveau mariage, ne bougeait pas de son palais de Ravenne, les armées de l'empereur Justinien, quittant Rome, occupèrent les places fortes de l'Etrurie."

c) Un cas intéressant apparaît dans les Romana, 121, où cum de coordination est construit avec le subjonctif imparfait, d'après le modèle de cum narratif: "Sed ubi, frustrato circa purpuratum eius ictu tenetur, ardentibus mox focis intulit manum terroremque geminat dolo. "En, ut scias", inquid" 69 "quem uirum effugeris: idem trecenti iurauimus." Cum inter ${ }^{70}$ inmane dictu hic interritus, ille trepidaret, tam-

Hormis l'exemple cité, on rencontre souvent cumque (voir, d'ailleurs, ci-dessus, l'exemple des Getica, 196). On trouve également indeque (Get., 178) et d'autres cas pareils.

60 diu multumque - formule classique.

61 pour penitus - hyperurbanisme; du même praessus pour pressus (Get., 212). En échange, penitens pour paenitens (Rom., 342) etc.

62 intrinsecus - adverbe utilisé en latin classique et (en latin) impérial. Ici, son sens est séparatif. On retrouve aussi, chez Jordanès, forinsecus (indiquant la direction), à petite distance de intrinsecus, dans la même phrase - Get., 220.

63 qui au lieu de quae (s'accordant avec aues, ou ciconiae).

64 gliba pour gleba. Voir aussi note 46.

65 pour pomoerium.

66 adque pour atque. Voir également set pour sed (Rom., 121) etc. De pareilles graphies apparaissent d'ailleurs chez Florus, qui influence souvent Jordanès. Pour ce qui est du remplacement de $t$ pour $d$, voir note 43.

67 fouit pour fouet (voir, pour la confusion des voyelles

68 on remarque l'emploi de l'ablatif à la place du locatif.

69 inquid pour inquit. Voir note 66.

70 cum inter au lieu de cum interim. Cfr. Getica. 195: “inter que" - avec la graphie erronée que pour 
quam manus regis arderet." (Cfr. Florus, 1, 4, 5-6, d'où ce passage est copié: "Sed ubi, frustrato circa purpuratum eius ictu tenetur, ardentibus focis inicit manum terroremque geminat dolo. "En, ut scias", inquit, "quem uirum effugeris, idem trecenti iurauimus." Cum interim - inmane dictuí - hic interritus, ille trepidaret, tamquam manus regis arderet.").

Une action (ou un état) simultanée et durative peut être aussi exprimée dans des propositions introduites par cum "uere temporale". Voir Getica, 22: "hi cum inopes uiuunt, ditissime uestiuntur." Il s'agit, en fait, d'un cum "temporel proprement dit" en contexte adversatif-concessif.

Pour ce qui est de $u b i, u t$, quando et quod introduisant des temporelles de simultanéité, voir le commentaire ci-dessous.

C) Les actions momentanées sont exprimées dans des subordonnées dont les conjonctions introductives ont le sens de: "comme", "quand", "au moment où". Il s'agit des conjonctions: cum, que les grammairiens appellent cum "déterminatif" ou cum "temporel proprement dit", quando et dum.

Assez rarement utilisé par Jordanès, cum temporel proprement dit apparaît presque uniquement dans les Romana. Dans les Getica on n'en trouve que peu d'exemples, voir Getica, 22 (l'exemple cité supra). Quant à l'exemple de Getica, 311, on pourrait dire qu'il s'agit plutôt d'un cum à valeur narrative que d'un cum "uere temporale" (voir le commentaire à la page 48 ).

Parmi les quinze exemples de cum suivi de l'indicatif existant dans Romana (abstraction faite de la locution cum mox), dans un cas il s'agit d'un cum situé à mi-chemin entre cum temporel proprement dit et cum narratif: "Sed cum subiectio omnium Maurorum facta est, Iubas rex ueneno hausto defecit...", Rom., 211 (construction différente de Rufus, 4 , sur lequel Jordanès a repris l'idée respective). A noter la position de la subordonnée dans l'exemple qu'on vient de citer.

C'est toujours le subjonctif de qualification que l'on eût dû employer dans l'exemple suivant, sans qu'on puisse parler de cum narratif, mais simplement de cum "uere temporale": "cuius quod gaudium fuit, cum Duellius ${ }^{71}$ imperator non contentus unius diei triumpho per uitam omnem, ubi a cena rediret, praelucere funalia et praecinere sibi tibias iussit, quasi cotidie triumpharet.", Rom., 165 (Ce passage est, en général, copié sur Florus, 1, 18, 10, à cette différence près que la pause plus grande entre "quod gaudium" et "cum" chez Florus rapproche la valeur de cum de celle de tum: "Cuius quod gaudium fuit: cum Duillius imperator, non contentus unius diei triumpho, per uitam omnes, ubi a cena rediret, praelucere funalia et praecinere sibi tibias iussit, quasi cotidie triumpharet."). Cfr. Jordanès: "Sed alligati miraculo quodam fuere, cum catenas morsibus et ore temptassent, cum offucandas in uicem fauces prebuissent. 72,

quae - au lieu de interim. Interim apparît pourtant chez Jordanès, voir Romana, 86, Getica, 244.

71 archaïque pour Duillius, ou, plus probablement, il s'agit de la confusion des voyelles $e$ et $i$ (voir notes 24,46 etc.). 
Rom., 224; voir également Romana, 164: "Proelii uero forma mirauilis ${ }^{73}$, cum illas caeleres $^{74}$ uolucresque hostium naues hae graues tardaeque conpraehenderent. ${ }^{75}$ " (passage copié sur Florus, 2, 2, 8).

En d'autres cas - parmi les quinze exemples construits avec l'indicatif des Roma$n a$ - il faut parler d'un cum à caractère causal bien marqué (voir Romana, 328), d'un cum adversatif-concessif (voir Romana, 299; Getica, 22) et d'un cum de coordination (accompagné de tamen - voir Romana, 136 et 209).

Cum "uere temporale" indique en général, chez Jordanès, des actions momentanées ou considérées comme telles. Le temps de la subordonnée est d'habitude le parfait de l'indicatif (voir Romana, 127, 139, 140, 193 etc.); le présent historique apparaît peu souvent (Romana, 152); dans un seul cas, c'est le futur antérieur qui est employé (Romana , 52). Dans la proposition corrélative, on retrouve le plus souvent leparfait de l'indicatif (voir Romana, 206 etc.); l'imparfait de l'indicatif est rarement utilisé (Romana, 127 et 152); le présent historique et le futur n'apparaissent qu'une seule fois chacun (voir Getica, 311 et, respectivement, Romana, 52); enfin, dans une seule proposition principale, le verbe (fuit) est sous-entendu (Romana, 193).

Cum temporel exprime, dans les oeuvres de Jordanès, soit:

I. la parfaite simultanéité entre l'action de la principale et l'action de la subordonnée, soit:

II. une antériorité récente par rapport à l'action de la principale, situation moins fréquente.

C'est, d'une part, le contexte et, d'autre part, les antécédents, ainsi que les temps employés dans les temporelles par rapport aux temps des principales, qui nous aident à déterminer la nature du rapport entre la subordonnée et sa principale.

Nous retrouvons ainsi, les situations suivantes:

I.

a) "Ludos forte celebrabat (ciuitas), cum adremigantes litore ${ }^{76}$ Romanam classem $^{77}$ uident..." Rom., 152. (Cfr. Florus, 1, 18, 4: "Ludos forte celebrabat, cum adremigantes litori Romanas classes inde uident...");

72 pour praebuissent.

73 pour mirabilis. La confusion entre les consonnes $b$ et $u$ est assez fréquente chez Jordanès.

74 au lieu de celeres.

75 pour comprehenderent. Quant à la forme con-du préfixe, voir aussi Romana, 360: conpraehensum; Romana, 363: conpescuit; Getica, 121: conperimus; Getica, 141: conperisset etc.

76 litore pour litori, voir les notes antérieures, concernant la confusion entre $e$ et $i$, confusion que favorise, ici, la position finale de la voyelle.

77 le singulier à la place du pluriel (voir le participe: adremigantes). Serait-ce une faute des copistes? Dans deux des plus précieux manuscrits apparaît: "Romana classe" (Codex Heidelbergensis - VIII siècle - et Codex Valenciennensis - IX ${ }^{\mathrm{e}}$ siècle). Cependant Florus emploie le pluriel: "Romanas classes". 
b) "Non fuit maior sub imperio Romano dies quam ille, cum duo omnium et antea et postea ducum maximi duces .... collatis comminus signis direxere aciem.", Rom., 206. (passage copié sur Florus, 2, 6, 58).

c) "Semel apud Anienem trucidati, cum singulari certamine Manlius aureum torquem barbaro inter spolia detraxit, unde et Torquatus est dictus.", Rom., 139 (passage copié sur Florus, 1, 13, 20). Voir aussi Romana, 140 (passage copié sur Florus, 1, $13,20)$. Comparer au type de temporelle introduit par quando: "A confusione ergo linguarum .... et usque natiuitatem Abrahae, quando et primus rex in mundo in gente regnabat Assyriorum Ninus..., fiunt anni DXLI sic.", Rom., 10.

II. - “... et cum se locus obtulerit, ad eum ordinem redeam", Rom., $52 .{ }^{78}$

Pour cum "uere temporale" les corrélatifs sont extrêmenent rares: un seul exemple de mox-Romana, 87.

Si cum "uere temporale" garde parfois chez Jordanès sa valeur et sa construction classiques, ce qu'il y a de surprenant, c'est sa faible fréquence: quando et dum remplacent en bas latin et chez Jordanès cum "temporale" (ou déterminatif). Dans la série des conjonctions temporelles spécifiques du latin cultivé à l'époque classique celles que Jordanès remplace par d'autres, dans une plus grande mesure, ce sont: cum, ut et priusquam (voir la discussion ci-dessous). Cependant, cum dans ses diverses acceptions temporelles se conserve mieux, chez Jordanès, que cum causal ou cum adversatif-concessif, ces dernières valeurs substituées, dans une mesures encore plus large, par d'autres conjonctions.

Evité par la langue littéraire à diverses époques (Térence, Varron, Salluste, César, Cicéron dans ses discours, Tacite, Pline le Jeune, Quintilien et d'autres), quando semble avoir été une conjonction temporelle particulièrement fréquente en latin vulgaire et familier tant à l'époque classique que dans les siècles suivants. ${ }^{79}$

Jordanès, tout comme d'autres auteurs tardifs (voir Vulgata, Luc., 4, 24-27; Peregrinatio Aetheriae, 4, 8; Augustin, Serm., 38, 5, 7; Orose, Hist., 2, 12, 2 al.; Grégoire de Tours, Hist. Franc.., 8, 31, etc.), emploie souvent la conjonction quando: nous en trouvons six exemples dans les Romana et dix dans les Getica. De ces seize exemples, dans deux cas il s'agit du quando itératif, dans deux autres - de quando à caractère causal et, une seule fois, de quando adversatif (qui équivaut à cum de coordination).

Quando itératif, de même qu'un exemple de quando explicatif-causal, sont construits avec le subjonctif (voir, pour quando itératif, le paragraphe concernant les subordonnées itératives). Dans les autres cas, c'est bien l'indicatif qui est employé.

78 Dans certains cas, il est difficile à dire si l'auteur envisage une action comme simultanée ou d'antériorité récente par rapport à l'action de la principale, s'il considère une action comme durative ou momentanée etc. En plus, les dérogations de Jordanès aux normes classiques embrouillent parfois l'interprétation des exemples.

79 A propos de la fréquence de quando chez Plaute, voir $\mathrm{T}$. Vasilesco, Curs de sintaxa istorica a limbii latine, Bucarest, 1962, p. 262. Quando est l'une des rares conjonctions temporelles latines que les langues romanes aient conservées, voir it.: quando, fr.: quand, esp.: cuando, port.: quando etc. En fait, quandos'est transmis dans toutes les langues romanes. 
Voici quels types d'exemples on rencontre dans les Romana:

a) Noe uero sexcentorum ${ }^{80}$ erat annorum, quando diluuium mundi crudelissima facinora expiauit.", Rom., 9;

b) "A confusione ergo linguarum ... et usque natiuitatem Abrahae, quando et primus rex in mundo in gente regnabat Assyriorum Ninus..., fiunt anni DXLI sic.", Rom., 10. Voir également Romana, 76: "Sub hoc item uicti Iudaei et sexaginta milia eorum caesa ab Anthioco ${ }^{81}$ rege Syriae, quando et ${ }^{82}$ pontifex magnus Onias."

La majeure partie des exemples des Romana appartiennent à ce dernier type, voir par. 51, 312, 367.

Pour ce qui est de Getica, non seulement quando y est plus fréquent, mais encore ses usages sont plus variés.

En dehors de l'usage que nous avons déjà mentionné dans les Romana - le type $b$ (deux exemples seulement de ce type dans les Getica, voir par. 104: "Defuncto tunc Decio Gallus et Volusianus regnum ${ }^{83}$ potiti sunt Romanorum, quando et postilens morbus .... faciem totius orbis foedauit..." - l'idée est reprise sur Jérôme, Chron. ad an. 2269, mais la construction grammaticale est différente; voir également Getica, 166), nous rencontrons quando à la place de cum inuersum: "Cui prouinciae tunc post Agrippam Oppius praeerat Sauinus ${ }^{84}$, Gothis autem Dorpaneus ${ }^{85}$ principatum agebat, quando bello commisso Gothi, Romanos deuictos, Oppii Sauini caput abscisum ${ }^{86}$, multa castella ... depraedarunt ${ }^{87}$., Get., 76. Vu l'importance que 1'historien accorde dans ses ouvrages aux succès militaires des Goths ${ }^{88}$, nous sommes portés à croire qu'il s'agit, ici, d'un quando inuersum. Mais il ne serait pas impossible d'interpréter ce quando comme un substitut de cum "uere temporale", dans une manière d'expression inadéquate (voir, d'ailleurs la note 78).

Quando apparaît également dans les Getica, avec son sens ancien, explicatif-temporel: "Eratque tunc in tribus his germanis contemplatio grata, quando mirabilis Thiu-

80 pour sescentorum (quant au phénomène inverse, voir Xerses-Romana, 62, Getica, 64; senis Romana, 33 etc.).

81 pour Antiocho.

82 Th. Mommsen (édition citée, p. 187) considère que, dans les exemples de ce type, et est superflu. Nous l'interprétons non pas comme superfétatoire, mais comme ajoutant une information en quelque sorte de moindre importance aux faits communiqués dans la régissante.

83 complément à l'accusastif, au lieu de l'ablatif. Potiri apparaît assez souvent chez Jordanès avec l'accusatif à la place de l'ablatif (notamment dans les Getica).

84 pour Sabinus. La confusion entre $u$ et $b$ est assez fréquente chez Jordanès, tant pour des noms propres que pour des noms communs, de même que pour des verbes etc. Voir aussi note 73.

85 Dorpaneus au lieu de Decebalus - Decebale.

86 caput abscisum - accusatif absolu, construction fréquente chez Jordanès.

87 la forme active (erronée) au lieu de la forme moyenne.

88 En fait, il s'agit ici des victoires remportées par les Gètes, ou, mieux, par les Géto-Daces, sur les Romains. A propos de la confusion fréquente entre Goths et Gètes dans les écrits de Jordanès, voir R. Iordache, La confusion "Gètes - Goths" dans "Getica" de Jordanès, op.cit.. 
dimer pro fratris Valamir ${ }^{89}$ militabat imperio, Valamir uero pro altero iubebat ${ }^{90}$ ornando, Vidimer seruire fratribus aestimabat ${ }^{\prime 91}$., Get., 253. Le passage est, dans ses grandes lignes, inspiré de Cassiodore, Var., 11, 1, mais il est très différent sous l'aspect de la construction grammaticale.

Pour l'usage ancien, explicatif-temporel de quando, voir aussi Getica, 214.

C'est toujours dans les Getica que l'on peut rencontrer des exemples de quando itératif, quando explicatif-causal et quando de coordination (exemples mentionnés pages 51).

Jordanès emploie quando soit pour exprimer des actions momentanées, soit pour des actions duratives. L'imparfait de l'indicatif apparaît dans les exemples cités Romana, 10, Getica, 253, et aussi Getica, 214. Le présent de l'indicatif, pour des actions et des états duratifs, apparaît dans une subordonnée explicative-causale (voir Getica, 52) et dans la proposition introduite par quando de coordination (Getica, 189). L'imparfait du subjonctif désigne une action durative dans la subordonnée explicative-causale des Getica, 213.

La concordance des temps est respectée. Dans la principale, on retrouve le plus souvent l'imparfait de l'indicatif (voir Romana, 9, 51, 367, Getica, 76, 213, 253), parfois le parfait de l'indicatif (Romana, 76, Getica, 104, 166), à deux reprises - le présent (non-historique) de l'indicatif (Romana, 10, Getica, 52), et, dans un seul exemple, l'imparfait du subjonctif (puisque la principale est une subordonnée consécutive - Getica, 214). Dans la subordonnée, c'est surtout le parfait de l'indicatif qui est de règle (voir Romana, 51, 367; Getica, 76, 104 etc.) - cfr. la fréquence de l'indicatif parfait dans les subordonnées introduites par cum "uere temporale" (voir ci-dessus, p. 50). Pour les autres temps utilisés dans les subordonnées introduites par quando, voir l'alinéa précédent.

Les corrélatifs sont rares: c'est seulement l'adverbe tunc, utilisé dans les Getica, 76 et 253 (voir les exemples cités). Aucune des subordonnées introduites par quando dans les Romana ne présente de corrélatif.

Le choix de tunc n'est nullement fortuit: tunc est un adverbe largement utilisé par Jordanès, tout comme dans le latin vulgaire de l'époque tardive ${ }^{92}$ (chez Jordanès, l'adverbe tunc remplace, en grande mesure, tum). L'adverbe tunc apparaît en tant que corrélatif aussi pour $u b i$.

89 forme invariable ici, à la place du génitif Valameris.

90 iubebat au lieu de Laborabat (ou: uiuebat).

91 aestimabat avec le sens de "magni aestimare".

92 Tunc, simple ou renforcé par la particule -ce et précédé de in, ad ou ex s'est transmis dans grand nombre de langues romanes (voir Fr. Diez, Etymologisches Wörterbuch der Romanischen Sprachen, Bonn, 1869, $3^{\mathrm{e}}$ éd., vol. II, p. 126; Adolf Zauner, Altspanisches Elementarbuch, Heidelberg, 1921, $2^{\mathrm{e}}$ éd., par. 147; J. J. Nunes, Compendio de Gramatica Historica Portuguesa, Lisboa, 1930, 2 éd., p. 358 et d'autres encore). 
Bien que moins fréquent que quando, dum se substitue également à cum "temporel" (ou "uere temporale").

Dum “temporel proprement dit" est attesté à partir du premier siècle de notre ère, mais il se peut qu'il fût déjà utilisé dans les siècles antérieurs. Dum - forme d'instrumental (tout comme cum) - a une évolution sémantique proche de celle de cum; le sens "lorsque" est vraisemblablement apparu à l'époque préclassique déjà. Dum "temporel proprement dit"est utilisé tant pour des actions momentanées que pour des actions duratives". Primitivement, dum "uere temporale" apparaît pour des actions momentanées ou considérées comme telles. A l'époque postclassique, dum est construit avec l'indicatif - d'habitude présent et futur antérieur, parfois parfait et futur (voir Rutilius Lupus, 1, 16; Celse, 5, 28, 1 B; Columelle, 7, 8, 7-parfait de l'indicatif; ibid., 7, 3, 19 - futur antérieur; voir ensuite Arnobe, Ad Greg., 17, p. 44, 8; Palladius, 2, 8; Benoît de Nursie, Reg. Monach., chap. 48,16 etc. etc.) ${ }^{94}$.

Nous soulignons que dum "temporel proprement dit" est inusité en latin cultivé, à l'époque classique.

Cette acception de $d u m$ - fréquente en latin familier et vulgaire à l'époque tardive - est rare chez Jordanès.

Dans certains passages, le sens de dum est à mi-chemin entre le sens "par le fait que" et le sens "lorsque". Voir Getica, 273: "Quiescente uero tandem Hunnorum gente a Gothis Hunumundus ${ }^{95}$ Suauorum ${ }^{96}$ dux $d u m$ ad depraedandas Dalmatias transit, armenta Gothorum in campis errantia depraedauit ${ }^{97}$...". Voici la traduction de ce fragment: "Mais, quand enfin les Huns jouissaient de paix de la part des Goths, Hunimundus, le roi des Suèves, pilla - lorsqu'il passa (= en passant) dans les Dalmaties pour les mettre à sac - les troupeaux des Goths qui erraient dans les champs...". Voir également Romana, 358: "Contra ${ }^{98}$ quem dum Hypatius nepus ${ }^{99}$ Caesaris cum exercitu numeroso $^{100}$ pugnaturus $^{101}$ egreditur, ante ${ }^{102}$ ab Hunnis auxiliaribus capitur et Vita-

93 contrairement à l'opinion de certains chercheurs qui prétendent que dum avec la valeur de cum "temporel proprement dit" est utilisé uniquement pour des actions momentanées (voir Thesaurus linguae Latinae, vol. V - 1, op.cit., p. 2218, 1. 39).

94 Pour d'autres exemples, voir Thesaurus linguae Latinae. vol. V - 1, op.cit., p. 2218.

95 La forme courante est Hunimundus (c'est elle qui est plus fréquente chez Jordanès aussi; d'aileurs, même pour ce paragraphe - Getica, 273, certains manuscrits précieux indiquent la forme Hunimundus - voir Codex Valenciennensis, IX siècle et Codex Breslaviensis, $\mathrm{XI}^{\mathrm{e}}$ siècle.)

96 pour Sueborum, ou Sueuorum.

97 une fois de plus, forme active du verbe depraedari (voir aussi note 87).

98 On remarque l'emploi de la préposition contra à la place de in. La préposition contra apparaît fréquemment chez Jordanès, suivant d'ailleurs la tradition vulgaire.

99 nepus pour nepos. Ce nom présente toujours, chez Jordanès, la graphie nepus - au nominatif. La confusion entre $o$ et $u$ est relativement fréquente dans les écrits de Jordanès.

100 Numerosus au sens de “abondant", “nombreux", appartient à l'époque impériale (voir aussi Romana, 363: "numerosus ruit exercitus"; Romana, 366: "numerosos milites"). Dans les Getica on rencontre aussi le substantif numerositas (par. 119 et 181). 
liano .... turpiter uenditur, antequam aperto proelio ... sese inimicum ostenderet.", fragment dont la traduction est: "Lorsque Hypatius, neveu de l'empereur, s'avança (= s' avançant) pour combattre celui-là, à la tête d'une armée nombreuse, il fut capturé par les auxiliaires huns et fut honteusement vendu à Vitalien, avant même d'avoir pu montrer dans un combat ouvert combien il haïssait cet ennemi."

A côté du présent historique, on peut aussi rencontrer le parfait de généralisation - il s'agit, en fait, d'une subordonnée explicative itérative: "Sic humana fragilitas, dum suspicionibus occurrit, magna plerumque agenda ${ }^{103}$ rerum occasione ${ }^{104}$ intercepit", Get., 217.

$\mathrm{Si}$, dans certains cas, le sens de dum oscillait entre "par le fait que" et "quand", il y a des situations où le sens "quand" et même le sens "au moment (exact) où" sont indubitables. Voir Romana, 351: "Qui parens praeceptis reginae, dum auidus ferit in capite $^{105}$ ense, non ceruices, ut cupiebat, sed aurem illius amputauit." (l'idée de ce passage était inspirée de Marcellin, a. 484; la construction grammaticale appartient à Jordanès). Voir également Getica, 97, où dum est suivi du parfait de l'indicatif (coepit).

Les subordonnées qu'introduit dum "temporel proprement dit" précèdent, en général, la principale, mais il se peut qu'elles soient intercalées entre le sujet et le verbe de la principale.

Dum avec la valeur de cum "temporel proprement dit" est, chez Jordanès, plus rare que dum remplaçant cum narratif de simultanéité.

En tout cas, dum "uere temporale" est un peu plus fréquemment employé dans les Getica que dans les Romana.

Le rapport de simultanéité, de même que le rapport d'antériorité immédiate, sont rendus aussi par des propositions relatives proprement dites. Voici des exemples illustrant le rapport de simultanéité: "Dicineus ${ }^{106}$ uenit in Gothiam" ${ }^{107}$, quo tempore Romanorum Sylla potitus est principatum. ${ }^{108}$, Get., 67 . Voir également Getica, 174 etc.

Et voici des exemples d'antériorité immédiate: “.... uidens Valia Vandalos in suis finibus $^{109}$.... egressos et cuncta in praedas uastare, eo fere tempore, quo Hierius et

101 Le participe futur actif à valeur finale est caractéristique du latin familier et vulgaire.

102 ante - antécédent de la conjonction antequam. A propos de cet exemple, voir le paragraphe "Le rapport temporel de postériorité". Pour l'accumulation de marques, voir aussi le paragraphe "Le rapport temporel d'antériorité".

103 au lieu de la forme de génitif de la construction avec l'adjectif verbal - le gerundiuum.

104 forme d'accusatif où la finale - $m$ n'est pas marquée.

105 in capite au lieu de in caput.

106 Dicineus pour Decaeneus (ou Decaineos).

107 in Gothiam au lieu de in Daciam.

108 complément en accusatif à la place du génitif.

109 ablatifà la place de l'accusatif (le complément dépend de egressos). On remarque aussi l'adjectif suis placé avant le substantif - effet de style. 
Ardabures consules processissent ${ }^{110}$, nec mora ${ }^{111}$ mox $^{112}$ contra $^{113}$ eos mouit exercitum.", Get., 166. La traduction de ce fragment est: "Voyant que les Vandales étaient entrés dans ses terres et qu'ils saccageaient tout, à peu près dans la même période où Hiérus et Ardaburès étaient devenus consuls, Valia leva bientôt, sans tarder, une armée contre eux." Voir aussi Getica, 251: “... et tempus accederet, quo Valamer ${ }^{114}$ habitum repararet ${ }^{115}$ uirilem...".

La simultanéité, mais dans un sens plus large, peut être indiquée par des conjonctions itératives aussi. La proposition itérative - qui marque le fait que l'action de la principale se réalise toutes les fois que l'action de la subordonnée se répète - exprime toujours un rapport de simultanéité.

Les conjonctions itératives spécifiques: quotiens et quotienscumque manquent totalement dans les écrits de Jordanès. D'ailleurs, dans les textes influencés par le latin vulgaire elles pénètrent rarement. Les langues romanes n'ont pas hérité quotiens et quotienscumque.

L'indicatif - mode employé pour la répétition en latin préclassique et dans le latin cultivé de l'époque classique - est souvent remplacé par le subjonctif, un subjonctif dit "de répétition" qui, à la fin de l'époque républicaine, mais notamment à l'époque postclassique, est usuel après cum, quando, si, ubi, quotiens, ut quisque etc. (voir de tels exemples chez Cicéron, César, bien fréquemment chez Népos ${ }^{116}$, souvent chez Tite-Live $^{117}$, Velléius Paterculus, Tacite, Suétone ${ }^{118}$, Justin, Ammien, dans la Vulgate etc.). Le subjonctif "de répétition" avait été préparé, depuis des siècles, par les liens étroits qui unissaient l'idée de répétition à celle d'éventualité ${ }^{119}$. A ceux-ci s'ajoute,

110 subjonctif plus-que-parfait au lieu de l'indicatif plus-que-parfait.

111 nec mora au lieu de sine mora, ou statim. Nec mora apparaît dans d'autres passages aussi: Getica, 28 - "nes mora ilico ad gentem Spalorum adueniunt"; Getica, 65: "nec mora soluta acie"; Getica, 307; ibid., 309 etc.

Nec mora appartient, sans doute, au langage familier et vulgaire.

Chose remarquable, nec mora est combinée avec l'adverbe mox, ou avec ilico (pour ce dernier cas, voir l'exemple cité dans cette note même, un peu plus haut). De pareils pléonasmes apparaissent dans les Romana aussi (voir le paragraphe 163: "statim ac sine mora") et sont typiques du latin vulgaire.

112 Adverbe particulièrement fréquent chezJordanès (voir notre discussion là-dessus, dans le paragraphe concernant la conjonction mox).

113 contra à la place de in.

114 pour Valamir.

115 au lieu de pararet. Jordanès emploie assez souvent des verbes composés au lieu des verbes simples; relativement à ce sujet, voir Th. Mommsen, Index, op.cit., p. 182.

116 Chez Népos, la construction avec le subjonctif est dominante (voir A. Tovar, Gramtica histórica latina -Sintaxis, Madrid, 1946, p. 220, par. 381).

117 voir O. Riemann - E. Benoist, Remarques sur la langue de Tite-Live, XXIII-XXV, Paris, Hachette, $1904,8^{\mathrm{e}}$ éd., pp. 362-63, $\mathrm{n}^{\circ}$ 178. Voir également A. Tovar, op.cit., p. 220, par. 381.

118 Pour Tite-Live, Tacite, Suétone, voir R. Kühner - C. Stegmann, 2. Teil, 2. Bd., op.cit., pp. 206-07, par. 182, $8^{\circ}$; quant à Tite-Live, Tacite, Vulgate, voir Al. Ernout-Fr. Thomas, op.cit., p. 400, par. 389; pour Velléius, Tacite, voir R. Ochesanu, Istoria limbii romane, Bucarest, 1965, p. 327. 
très probablement, l'analogiè avec la construction de cum narratif ${ }^{120}$ (mentionnons, dans cet ordre d'idées, le choix de l'imparfait et du plus-que-parfait du subjonctif dans les subordonnées itératives - nous parlons, bien sûr, des auteurs classiques et de ceux qui, dans les siècles suivants, imitent les classiques.).

Les propositions itératives, assez rares chez Jordanès, sont introduites par $u b i$, quando et dum (énumérés par ordre de leur fréquence dans cet usage). Toutes ces trois conjonctions sont fréquentes en latin vulgaire à l'époque tardive. Il faut ajouter la conjonction $u t$ - un seul exemple.

La subordonnée itérative apparaît surtout dans les Getica. C'est toujours ici que l'on retrouve une plus grande variété de constructions.

Parmi les quatre conjonctions citées, seuls dum et $u t$ sont construits exclusivement avec l'indicatif - d'ailleurs, il n'en est qu'un seul exemple pour dum, Getica, 217 (que nous avons commenté pages 54-55) et un autre exemple pour ut, Getica, 70 (voir ci-dessous).

Après $u b i$ et quando, c'est le subjonctif qui est prédominant. Voir Getica, 200: "Reliqua autem, si dici fas est, turba regum diuersarumque nationum ductores ac si satellites notibus ${ }^{121}$ Attilae attendebant, et, ubi oculo annuisset, absque ${ }^{122}$ aliqua murmuratione cum timore et tremore ${ }^{123}$ unusquisque adstabat, aut certe, quod iussus fuerat $^{124}$, exequebatur ${ }^{125}$."

Le subjonctif précédé de ubi apparait aussi dans les Romana, 165 - nous renvoyons à la phrase citée page 49 (il s'agit d'un subjonctif imparfait); en fait, la construction est reprise sur Florus, 1, 18, 10. C'est toujours le subjonctif qui apparaît après $u b i$ dans les Getica, 165 (en fait le subjonctif parfait) et Getica, 119. Dans ce dernier exemple, le subjonctif parfait alterne avec l'indicatif.

Pour ce qui est de la conjonction quando, voir Getica, 70: "Qualis erat, rogo, uoluptas, ut uiri fortissimi, quando ab armis quantolumcumque ${ }^{126}$ uacassent, doctri-

119 Voir Al. Ernout - Fr. Thomas, op.cit., pp. 399-402, par. 389 et 390.

120 Voir R. Kühner - C. Stegmann, 2. T., 2. Bd., op.cit., pp. 206-07, par. 182, $8^{\circ}$.

121 notibus pour nutibus. Voir aussi Getica, 157: notu pour nutu. Cependant, dans le passage cité-Getica, 200 , on trouve la forme correcte: annuisset. La confusion entre les voyelles $o$ et $u$ est un phénomène relativement fréquent chez Jordanès.

122 absque apparaît trois fois chez Jordanès (et uniquement dans les Getica).

123 "cum timore et tremore" - exagération stylistique.

124 iussus fuerat au lieu de iussus erat. L'auxiliaire esse se trouve, parfois, chez Jordanès, au parfait au lieu d'être au présent, au plus-que-parfait au lieu de l'imparfait, au futur antérieur à la place du futur (voir, par exemple: fuerat erectus, Get., 100; fuerant adepti, Get., 65; fuisse exortos, Get., 38 etc.). Les formes de ce genre sont particulièrement fréquentes dans le latin vivant à l'époque tardive.

125 exequebatur, au lieu de exsequebatur. Voir aussi extingui, Rom., 265 etc.

126 quantolumcumque, pour quantulumcumque (voir, à propos de la confusion entre $o$ et $u$, nos notes antérieures). On constate, de plus, la préférence de Jordanès pour les diminutifs, voir aussi Romana, 177: quantulum (avec la valeur de aliquantulum; Getica, 63 et 172: aliquantulum etc. Le diminutif quantolum est mis en évidence (et compliqué) par la juxtaposition du suffixe -cumque. 
nis philosophicis inbuebantur ${ }^{127}$ ?". Deux questions se poseraient au sujet de l'exemple que nous venons de citer:

a) Est-ce que "uacassent" est un subjonctif “de répétition", ou bien s'agit-il d'une forme de subjonctif plus-que-parfait à valeur d'indicatif plus-que-parfait?;

b) Le plus-que-parfait utilisé ici a-t-il valeur de plus-que-parfait, ou bien substitue-t-il l'imparfait (du même mode)?

a) La forme "uacassent" (pour "uacauissent"), ainsi que l'utilisation fréquente chez Jordanès et en latin vulgaire, à l'époque tardive, du subjonctif plus-que-parfait à la place de l'indicatif plus-que-parfait ${ }^{128}$, plaiderait contre l'hypothèse d'un subjonctif de répétition. Cependant, puisque d'une part ce type de subjonctif prédomine, chez Jordanès, dans la subordonnée itérative et que, d'autre part, il apparaît assez souvent à un autre temps aussi, à savoir le parfait (du subjonctif) - voir ci-dessous - nous sommes penchés à croire qu'il s'agit en effet du subjonctif de répétition.

b) Ce subjonctif plus-que-parfait se substitue-t-il à l'imparfait du subjonctif, ou bien s'agit-il de marquer, par l'emploi du plus-que-parfait, un rapport d'antériorité?

On sait qu'en latin vulgaire, à la fin du $\mathrm{I}^{\mathrm{er}}$ siècle av. J. C., de même que dans les siècles suivants (et notamment durant les $\mathrm{III}^{\mathrm{e}}-\mathrm{V}^{\mathrm{e}}$ siècles de notre ère), l'imparfait du subjonctif est peu à peu remplacé par le plus-que-parfait du subjonctif ${ }^{129}$. Chez Jordanès aussi, mais rarement, le plus-que-parfait du subjonctif prend la place de l'imparfait du même mode (voir la subordonnée consécutive: “... ita sunt preliati, ut ... non remansissent...", Get., 276) ${ }^{130}$.

Mais puisque, d'une part, quando itératif suivi de l'indicatif est construit avec le parfait (à valeur de parfait - voir ci-dessous Getica, 193) et que le subjonctif parfait (à valeur de parfait) apparaît dans des subordonnées itératives introduites tant par quan$d o$, que - surtout - par ubi et puisque, d'autre part, l'imparfait du subjonctif fait réellement son apparition (en fait, une seule fois, dans une subordonnée construite avec

127 inbuebantur à la place de imbuebantur. Voir aussi la forme: inbueret, Get., 35. En dehors de la tendance que manifeste Jordanès à rendre leur forme originelle aux préfixes, on peut parler de son souci tout à fait spécial de conserver (ou restaurer) le préfixe in- (voir Getica, 199: inlustrabat, Getica, 197: inponitur etc.)

128 Pour ce qui est de l'utilisation du subjonctif plus-que-parfait à la place de.l'indicatif plus-que-parfait, voir R. Iordache, Observaciones sobre la subordinada causal en las obras de Jordanes, op.cit., p. 9 et note 13 etc. Voir également ci-dessus note 110. Fr. Werner, dans la dissertation mentionnée, ne remarque pas la présence de ce phénomène chez Jordanès.

129 Le plus-que-parfait du subjonctif employé à la place du subjonctif imparfait est courant dans le Bellum Africum (voir W. Meyer-Lübke, Die lateinische Sprache in den romanischen Ländern, Grundriss, I, 2, p. 451 sqq.). Pendant la période comprise entre le III $^{e}$ et le $\mathrm{V}^{\mathrm{e}}$ siècle, les attestations de ce genre sont de plus en plus nombreuses (voir H. Rönsch, Itala und Vulgata. Das Sprachidiom der urchristlichen Itala und der katolischen Vulgata unter Berücksichtigung der römischen Volkssprache, Marburg, 1875, $2^{\mathrm{e}}$ éd., p. 431; C. H. Grandgent, Introducción al latín vulgar, Madrid, 1928, p. 95, et d'autres encore).

130 On trouve également, chez Jordanès, la situation inverse, à savoir l'utilisation du subjonctif imparfait à la place du subjonctif plus-que-parfait (voir Getica, 210). Voir aussi notre commentaire infra. 
ubi-Romana, 165), nous sommes penchés à considérer que, dans la subordonnée en discussion, le plus-que-parfait indique l'antériorité, d'après le modèle de cum narratif $^{131}$.

Un autre aspect intéressant que met en évidence l'étude de la temporelle itérative, c'est l'alternance, dans la même phrase, du subjonctif de répétition avec l'indicatif de répétition ${ }^{132}$. De telles situations apparaissent tant après quando qu'après ubi. Voir Getica, 193: "Probatum est humanum genus regibus uiuere, quando unius mentis insano impetu strages sit facta populorum et arbitrio superbi regis momento defecit quod tot saeculis natura progenuit. ("On a pu constater que l'espèce humaine vit pour les rois, toutes les fois que la fureur d'un seul fou provoque le massacre des peuples et chaque fois que le caprice d'un roi orgueilleux renverse dans un instant tout ce que la nature a mis tant de siècles à produire.").

Voir aussi Getica, 119: "Sed nihil ualet multitudo inbellium ${ }^{133}$, praesertim ubi et deus permittit et multitudo armata aduenerit." L'indicatif, dans la subordonnée de la dernière phrase, est reclamé aussi par le contenu de la proposition "Deus permittit" (vérité généralement valable).

L'emploi, dans la subordonnée itérative, de l'indicatif est restreint. Vbi apparaît quatre fois, accompagné de cinq verbes dont un seul à l'indicatif. Quando apparaît deux fois accompagné de trois verbes dont un seul à l'indicatif. Dum et ut itératifs sont employés une seule fois, mais avec l'indicatif. Dans les cas de $u b i$ et notamment de quando, l'indicatif n'est pas indépendant, mais il forme un couple avec le subjonctif.

La situation statistique des temps se présente de la manière suivante: subjonctif plus-que-parfait - deux verbes; subjonctif imparfait - un verbe; subjonctif parfait trois verbes; parfait de l'indicatif - deux verbes; indicatif présent - un verbe. Et voici maintenant l'évidence numérique des temps et des modes pour chaque conjonction:

$u b i-1$ subjonctif plus-que-parfait;

1 subjonctif imparfait;

2 subjonctifs parfait;

1 indicatif présent.

quando - 1 subjonctif plus-que-parfait;

1 subjonctif parfait;

1 indicatif parfait.

dum -1 indicatif parfait.

$u t-1$ indicatif imparfait.

131 Fr. Werner, op.cit., p. 87, soutient que, dans cet exemple, le plus-que-parfait du subjonctif se substitue à l'imparfait du subjonctif.

132 Pour l'alternance du subjonctif de répétition avec l'indicatif de répétition, voir Cicéron, Verr., 4, 48. César lui-même rattache parfois (dans De bello Gallico et De bello ciuili) le subjonctif de répétition à l'indicatif de répétition.

133 inbellium, pour imbellium (génitif pluriel). Voir aussi note 127. 
On peut observer que l'imparfait du subjonctif et le présent de l'indicatif n'apparaissent qu'après $u b i$, l'imparfait de l'indicatif fait son apparition après $u t$, tandis que quando, qui subit en plus grande mesure l'influence de la conjonction cum, est construit avec des temps indiquant une antériorité évidente (voir les exemples cités Getica, 70 et 193).

Autre remarque: le parfait du subjonctif est la forme la plus courante chez Jordanès dans la subordonnée itérative. Evité chez les écrivains classiques et chez les grands prosateurs du latin postclassique (voir, ci-dessus, p. 56), le subjonctif parfait n'apparaît qu'accidentellement dans les ouvrages des auteurs qui imitent, à l'époque tardive, le style classique (voir, par exemple, Avit ${ }^{134}$ ). Cependant, chez Grégoire de Tours, l'emploi du subjonctif parfait dans la subordonnée itérative introduite par cum, est un trait caractéristique $\mathrm{e}^{135}$. Chez Jordanès non seulement la proportion de l'emploi du parfait du subjonctif est grande, mais, parfois, l'utilisation de ce temps contrevient aux règles de la concordance des temps (règles que Jordanès observe en général). Voir Getica, 165: “.... ita conuenit pacisci, ut Placidiam sororem principis redderet suaque solacia ${ }^{136}$ Romanae rei publicae, ubi usus exegerit, non denegaret." (idée probablement reprise sur Orose, 7, 43, 12. La construction grammaticale appartient néanmoins à Jordanès). Dans cette phrase, il eût été plus convenable d'employer le plus-que-parfait du subjonctif.

(À suivre.)

134 Voir H. Goelzer, Le latin de S. Avit, Paris, 1909, p. 340, note 1 et p. 340, $2^{\circ}$ (par. 212).

135 Voir M. Bonnet, Le latin de Grégoire de Tours, Paris, 1880, pp. 684-85.

136 solacia avec la valeur du substantif auxiliares (avec la même acception dans les Romana, 223, Getica, 110 etc.). 